Rev. Int. Contam. Ambie. 37, 345-355, 2021

https://doi.org/10.20937/RICA.53719

\title{
RESPUESTA DE LA GLADIOLA A LA APLICACIÓN DE BIOFERTILIZANTES Y ABONO ORGÁNICO
}

Gladiola's response to the application of biofertilizers and organic manure

\section{Evelia CRUZ-RUIZ ${ }^{1 *}$, Araceli CRUZ-RUIZ ${ }^{2}$, Rodolfo SERRATO-CUEVAS ${ }^{1}$ y Martín RUBÍ-ARRIAGA ${ }^{1}$}

\author{
${ }^{1}$ Facultad de Ciencias Agrícolas, Universidad Autónoma del Estado de México, Campus Universitario el Ce- \\ rrillo, Piedras Blancas, 50200 Toluca, Estado de México, México. \\ ${ }^{2}$ Facultad de Ciencias, Universidad Autónoma del Estado de México, Campus Universitario el Cerrillo, Piedras \\ Blancas, 50200 Toluca, Estado de México, México.
}

*Autora para correspondencia: ecruzruiz@yahoo.com.mx

(Recibido: agosto de 2019; aceptado: julio de 2020)

Palabras clave: fertilización combinada, microorganismos del suelo.

\begin{abstract}
RESUMEN
La gladiola es uno de los principales cultivos ornamentales de México, en cuya producción se usan fertilizantes químicos excesivos que pueden ocasionar problemas de salud y contaminación ambiental. Los abonos orgánicos y los biofertilizantes aumentan la eficiencia de los fertilizantes de síntesis química, incrementan la productividad de los cultivos, mejoran la calidad del suelo, preservan la salud humana y conservan el ambiente. En el contexto sostenible, el uso de biofertilizantes y abonos orgánicos en ornamentales es una alternativa viable porque es amigable con el hombre y el ambiente. Para analizar los efectos de Bacillus subtilis, Glomus fasciculatum, fertilización orgánica e inorgánica, y sus interacciones con la productividad de cormos y espiga floral en gladiola (Gladiolus grandiflorus) variedad Borrega roja, se estableció este trabajo a cielo abierto en condiciones de temporal en el Valle de Toluca. Se evaluaron 12 tratamientos producto de la combinación de Glomus fasciculatum $\left(\mathrm{A}_{\mathrm{m}}\right)$, Bacillus subtilis $\left(\mathrm{A}_{\mathrm{b}}\right)$, Glomus fasciculatum + Bacillus subtilis $\left(\mathrm{A}_{\mathrm{m}+\mathrm{b}}\right)$, abono orgánico $\left(\mathrm{B}_{\mathrm{v}}\right) \mathrm{y}$ fertilizante inorgánico $\left(\mathrm{B}_{\mathrm{i}}\right)$. G. fasciculatum + B. subtilis + abono orgánico favorecieron significativamente el diámetro del tallo floral y su peso seco, además de incrementar en $27 \%$ el diámetro del cormo y en $100 \%$ su peso; también afectaron el número de cormillos en el $77 \%$ y su peso en el $90 \%$. Estos resultados evidencian las ventajas del uso combinado de biofertilizantes y fertilizantes orgánicos en gladiola.
\end{abstract}

Key words: combined fertilization, soil microorganisms.

\begin{abstract}
Gladiola is one of the main ornamental crops in Mexico; however, excess chemical fertilizers are used in its production, which can cause health problems and environmental pollution. Organic fertilizers and biofertilizers increase the efficiency of chemical synthesis fertilizers, increase crop productivity, improve soil quality, preserve human health and preserve the environment. In the sustainable context, the use of biofertilizers
\end{abstract}


and organic fertilizers in ornamentals is a viable alternative because it is friendly with men and environment. To analyze the effects of Bacillus subtilis, Glomus fasciculatum, organic and inorganic fertilization and their interactions on the productivity of corms and flower spike in gladiola (Gladiolus grandiflorus) red sheep variety, a field experiment was established under temporary conditions in the Toluca Valley. Twelve treatments were evaluated as a result of the combination of Glomus fasciculatum (Am), Bacillus subtilis (Ab), Glomus fasciculatum + Bacillus subtilis (Am + b), organic fertilizer (Bv) and inorganic fertilizer (Bi). G. fasciculatum + B. subtilis + organic fertilizer significantly favored the floral stem diameter as well as the stem dry weight; also, the corm diameter increased $27 \%$, and its weight $100 \%$; they also affected the number of cornels in $77 \%$ and their weight in $90 \%$. These results show the advantages of the combined use of biofertilizers and organic fertilizers in gladiola.

\section{INTRODUCCIÓN}

La gladiola (Gladiolus grandiflorus Hort.), nativa del mediterráneo, África meridional y central, se propaga por cormos y se cultiva como flor de corte (Schwab et al. 2015). Su flor es apreciada por la diversidad de colores (Cantor y Tolety 2011). En México se siembran casi 22700 ha de gladiolas, y hay una gran demanda de esta flor todo el año. En 2017 sólo se sembraron 4606 ha (SIAP 2017). En flores de corte se emplean grandes cantidades de fertilizantes y agroquímicos, cuyo uso indiscriminado destruye la capa de ozono y aumenta el calentamiento global y la acidificación del suelo (Sahle y Potting 2013). En aguas subterráneas, estos productos causan toxicidad en humanos, y en aguas superficiales el nitrógeno y el fósforo dan lugar a la eutrofización que trastorna el equilibrio ecológico (Sharma et al. 2019).

Actualmente hay una creciente preocupación por la sostenibilidad ambiental, social y económica (Pullman et al. 2009); como resultado, ha surgido la horticultura sostenible para apoyar y mejorar interacciones biológicas. La sostenibilidad se logra cuando en la producción se aprovechan las relaciones biológicas que se producen naturalmente (Granatstein y Kupferman 2008). La preocupación por un mejor ambiente genera la necesidad de desarrollar estrategias sostenibles, como la reducción del uso de agroquímicos. En este contexto, el uso de indicadores para evaluar el efecto de las estrategias implementadas en la calidad del suelo es primordial (Astier et al. 2002, D'Hose et al. 2014). Las funciones que desempeñan los microorganismos del suelo tienen efectos directos e indirectos sobre el crecimiento y la calidad del cultivo, la calidad de los ciclos de nutrientes y la sostenibilidad de la productividad (Roger-Estrade et al. 2010).

Los biofertilizantes son productos complejos elaborados con microorganismos para mejorar la fertilidad del suelo y la productividad de los culti- vos. Ayudan a la asimilación de nutrimentos a través de procesos naturales de fijación de nitrógeno, solubilizan el fósforo y estimulan el crecimiento de las plantas mediante la síntesis de sustancias promotoras del crecimiento. Entre los microorganismos benéficos se encuentran los hongos micorrízicos arbusculares (HMA), integrantes de la simbiosis mutualista más común entre plantas y hongos del phylum Glomeromycota. Estos hongos desempeñan un papel clave en la nutrición y el incremento del rendimiento de los cultivos, debido a su capacidad para mejorar la absorción de agua y nutrientes minerales como el fosfato, nitrógeno y algunos microelementos en la planta huésped (Lee et al. 2013). En este proceso se transfiere al hongo hasta el $20 \%$ del carbono que fija (Parniske 2008). Los HMA desempeñan un papel vital en el ecosistema, como el crecimiento y la productividad de las plantas, ya que proporcionan nutrientes a éstas y mejoran el aprovechamiento del agua y la estructura del suelo, con lo que ayudan a mejorar la sostenibilidad de los ecosistemas (Smith y Read 2008). Los HMA son el componente principal de la microbiota del suelo en la mayoría de los ecosistemas y pueden usarse como indicadores sensibles de la calidad ecológica del suelo, porque responden a variaciones en el ambiente (Verbruggen et al. 2012).

Las especies de Bacillus son un tipo principal de rizobacterias que pueden formar esporas capaces de sobrevivir en el suelo durante un largo periodo de tiempo en condiciones ambientales adversas (Hashem et al. 2019). A través de la biofertilización, las poblaciones de Bacillus mejoran la biodisponibilidad de compuestos esenciales y aumentan el suministro de nutrientes minerales a la planta huésped. La producción de fitohormonas, como las auxinas, estimula la proliferación de raíces y la absorción de nutrientes. Cepas de B. subtilis productoras de citoquininas tienen un efecto beneficioso sobre el crecimiento de las plantas (Pérez-García y Romero 2011). 
López-Valdes et al. (2011) observaron un efecto positivo temporal sobre el crecimiento de las plantas al inocular con B. subtilis semillas de girasol (Helianthus annuus L.); la longitud de la raíz, su peso fresco y seco fueron significativamente mayores en las plantas inoculadas.

Alam et al. (2011) aplicaron HMA solos y en combinación con B. subtilis en geranio (Pelargonium graveolens), con lo que aumentaron el rendimiento de esta planta en 49.4 y $59.5 \%$, respectivamente; la producción total de aceite también aumentó de manera significativa debido a una mayor cantidad de biomasa. $B$. subtilis tiene un efecto sinérgico con HMA sobre el crecimiento de las plantas; la aplicación combinada de ambos resulta en mayor promoción del crecimiento de las plantas, aumento de la producción de enzimas y antioxidantes, solubilización de fósforo, actividad de biocontrol de enfermedades, nodulación de raíces y fijación de nitrógeno (Hashem et al. 2019).

El manejo integrado de nutrientes con la aplicación de biofertilizantes mejora la fertilidad del suelo; su aplicación aumenta la disponibilidad de nutrientes para las plantas y mejora su capacidad de crecimiento y rendimiento. Con el uso de biofertilizantes se puede disminuir la dosis de fertilizantes químicos y aumentar el rendimiento del cultivo entre 10 y $25 \%$ (Kholkute 2013).

Con el uso de rizobacterias, Shanmugam et al. (2011) incrementaron la longitud de la espiga (58.5\%) y la producción de cormo (27.4\%) en gladiolas, y disminuyeron la incidencia de enfermedades en el cormo. Con el uso de biofertilizantes y formulaciones comerciales se mejoraron algunos parámetros cualitativos y cuantitativos y se incrementó la fertilidad del suelo (Baskaran et al. 2014). Qasim et al. (2014) investigaron el efecto de la inoculación microbiana sobre el crecimiento y producción de gladiola en condiciones de campo y concluyeron que, con ésta, se facilita la absorción de nutrientes y se producen plantas de calidad superior. Da Silva et al. (2017) aislaron cepas bacterianas de la rizosfera y raíces de geranio de olor (Pelargonium graveolens), evaluaron algunas características promotoras del crecimiento en las plantas y el antagonismo contra Colletotrichum acutatum; identificaron que varias cepas de Bacillus tienen actividad antagonista in vitro contra $C$. acutatum y fueron capaces de producir sideróforos, mineralizar fosfato orgánico, solubilizar fosfato inorgánico y/o producir compuestos indólicos.

Dipanjali et al. (2018) favorecieron el crecimiento de gladiola y tuvieron rendimientos máximos con la aplicación de estiércol y biofertilizantes. Parlakova et al. (2019) observaron un incremento en la altura de la planta con bacterias promotoras del crecimiento y vermicomposta. En petunia (Petunia hybrida), la presencia de tres hongos diferentes originó efectos positivos (Hayek et al. 2012). Püschel et al. (2014) probaron el efecto de la inoculación de ocho plantas ornamentales con HMA y encontraron un aumento en el número de flores, en el tamaño de la flor, en el número de hojas y en la longitud de la planta. Sharma et al. (2017) determinaron el efecto de un abono orgánico adicionado con un consorcio de microorganismos, aplicado como única fuente de nutrientes o combinado con el $50 \%$ de la dosis recomendada de fertilizante químico, en el crecimiento de caléndula (Calendula officinalis); los parámetros físicos y químicos, así como las actividades enzimáticas del suelo, mejoraron con el aumento en la aplicación de composta.

Manoly et al. (2008) estudiaron el efecto de biogen + fosforina + levadura seca activa en Dahlia pinnata y detectaron un incremento significativo en el número de flores (57\%), longitud del tallo (24\%) y diámetro del tallo (17.6\%).

Satapathy et al. (2016) incrementaron la longitud de la espiga $(70.53 \mathrm{~cm})$, la longitud del raquis $(55.55 \mathrm{~cm})$ y el número de flósculos por espiga (13.12) en gladiola mediante la aplicación de la dosis recomendada de fertilizantes químicos al 75\% (100:50:60 kg de NPK/ha) en combinación con vermicomposta $(5 \mathrm{t} / \mathrm{ha})$ y biofertilizantes $(10 \mathrm{~kg} / \mathrm{ha})$ (Azospirillum y bacterias solubilizadoras de fósforo).

En el contexto. anterior el objetivo principal del presente trabajo fue analizar los efectos de Bacillus subtilis, Glomus fasciculatum, fertilización orgánica e inorgánica y sus interacciones sobre la calidad comercial de espigas florales y cormos de Gladiolus grandiflorus.

\section{MATERIALES Y MÉTODOS}

\section{Descripción del sitio experimental}

Esta investigación se realizó de agosto a diciembre de 2017 en el Campus Universitario El Cerrillo de la Facultad de Ciencias Agrícolas (FCA) de la Universidad Autónoma del Estado de México (UAEM), localizado a $19^{\circ} 26^{\prime} 00^{\prime \prime} \mathrm{N}$ y $99^{\circ} 43^{\prime} 00^{\prime}$ ' O, a 2656 $\mathrm{msnm}$. El clima predominante es templado subhúmedo con lluvias en verano $\left(\mathrm{C}\left[\mathrm{w}_{2}\right][\mathrm{w}] \mathrm{bi}\right)$ y temperatura media anual de $12.6^{\circ} \mathrm{C}$ (García 2004).

\section{Características del suelo}

Antes del establecimiento del cultivo, se obtuvieron muestras de suelo a una profundidad de $0-15 \mathrm{~cm}$. Con ellas se obtuvo una muestra compuesta que fue 
secada a la sombra a temperatura ambiente, tamizada y guardada en bolsas de plástico para su posterior análisis. Los análisis físicos y químicos del suelo se realizaron en el Laboratorio de Edafología de la FCA-UAEM. Las características físicas determinadas fueron: contenido de humedad por gravimetría, capacidad de retención de agua (CRA), textura (método de Bouyoucos), densidad aparente (método de la probeta), $\mathrm{pH}$ a partir de una suspensión suelo:agua en proporción $1: 2.5$, contenido de materia orgánica (método AS-07, NOM-021-RECNAT-2000), nitrógeno total (Kjeldahl) y conductividad eléctrica en extracto 1:2 suelo:agua determinada con un conductímetro (Cuadro I).

\section{Establecimiento del cultivo}

Se utilizaron cormos comerciales de gladiola de 3 $\mathrm{cm}$ de diámetro, variedad borrega roja previamente vernalizados. Los cormos fueron desinfectados un día antes de la plantación con $0.5 \mathrm{~mL} / \mathrm{L}$ de Procloraz (Sportak $45 \mathrm{CE}$ ) durante $10 \mathrm{~min}$. Luego se escurrieron y dejaron secar. La plantación se realizó a una profundidad de $7 \mathrm{~cm}$ en lotes experimentales constituidos por cinco surcos con separación de 0.80 $\mathrm{m}$ y $5 \mathrm{~m}$ de largo.

En el experimento se evaluaron los siguientes 12 tratamientos: G. fasciculatum $\left(\mathrm{A}_{\mathrm{m}}\right)$ : inóculo constituido por suelo limoso con 73 esporas/g de G. fasciculatum y fragmentos de raíz con $50 \%$ de colonización; $B$. subtilis $\left(\mathrm{A}_{\mathrm{b}}\right)$ : suspensión con $1 \times 10^{7} \mathrm{ufc} / \mathrm{mL}$ de $B$. subtilis BEB-1Sbs (BS-13) (absorbancia de 0.1 a $535 \mathrm{~nm}$ ); coinoculación con G. fasciculatum y B. subtilis $\left(\mathrm{A}_{\mathrm{m}+\mathrm{b}}\right)$; y un tratamiento testigo; cada uno de ellos considero las variantes sin fertilizar, fertilizante químico $\left(\mathrm{B}_{\mathrm{i}}\right)$ : urea, superfosfato de calcio triple, cloruro de potasio y sulfato de magnesio; y abono orgánico $(\mathrm{Bv})$ : biosólidos de la producción de levaduras con el siguiente contenido nutrimental: $55 \%$ de materia orgánica, $3.5 \%$ de $\mathrm{N}, 3.5 \mathrm{mg} / \mathrm{kg}$ de $\mathrm{P}, 1.7 \mathrm{mg} / \mathrm{kg}$ de $\mathrm{K}, 6.0 \mathrm{mg} / \mathrm{kg}$ Ca y $1.0 \mathrm{mg} / \mathrm{kg}$ de $\mathrm{Mg}$ y $\mathrm{pH} 8.0$.
La asignación de los tratamientos a las unidades experimentales se hizo con un diseño completamente al azar con tres repeticiones. En el cuadro II se muestran los tratamientos considerados.

Al momento de la plantación se aplicó el $50 \%$ de la dosis de fertilizantes y 30 días después se aplicó el 50 \% restante (González-Pérez et al. 2011, ValdezAguilar et al. 2015). Se emplearon 5 g por planta de la cepa de micorriza arbuscular ( $G$. fasciculatum) y $2 \mathrm{~mL}$ de $B$. subtillis $\left(1 \times 10^{7} \mathrm{ufc} / \mathrm{mL}\right)$. La aplicación se realizó a los 30 y 60 días de la plantación. El experimento se estableció a cielo abierto en condiciones de temporal, con temperatura y precipitación media durante el ciclo de cultivo de $12{ }^{\circ} \mathrm{C}$ y $300 \mathrm{~mm}$, respectivamente.

\section{Variables de crecimiento}

Las evaluaciones del crecimiento y rendimiento de la gladiola se realizaron en la madurez comercial (120 días). De cada parcela experimental fueron seleccionadas 10 plantas con competencia completa en los tres surcos centrales y con ellas se realizó el registro de datos. Las plantas seleccionadas fueron cosechadas y transportadas al laboratorio, donde se eliminaron los restos de suelo, se lavaron con agua destilada, se eliminó el exceso de agua y fueron secadas sobre papel. Se evaluaron los siguientes elementos: número de hojas, número de flores, longitud de la espiga floral, longitud del tallo floral, diámetro del tallo floral, peso fresco y peso seco, de acuerdo con la técnica sugerida por Rubí et al. (2009). El contenido de clorofila se estimó en la etapa vegetativa, 70 días después de la siembra, en las hojas 3, 4 y 5 con un medidor portátil SPAD-502 (Konica Minolta, Osaka, Japón). Los cormos se recogieron del campo 50 días después de la cosecha, se eliminaron los residuos de suelo y se realizó la toma de datos.

\section{Análisis estadístico}

Las características de las plantas se evaluaron estadísticamente por análisis de varianza y posteriormente

CUADRO I. CARACTERÍSTICAS FÍSICAS Y QUÍMICAS DEL SUELO EN QUE SE ESTABLECIÓ EL EXPERIMENTO.

\begin{tabular}{cccccccccccc}
\hline Propiedad & $\begin{array}{c}\text { Dap } \\
\left(\mathrm{gcm}^{-3}\right)\end{array}$ & $\begin{array}{c}\text { CRA } \\
(\%)\end{array}$ & $\begin{array}{c}\text { Clase } \\
\text { textural }\end{array}$ & $\mathrm{pH}$ & $\begin{array}{c}\mathrm{CE} \\
(\mathrm{dS} / \mathrm{m})\end{array}$ & $\begin{array}{c}\mathrm{Ca}^{2+} \\
(\mathrm{meq} / 100 \mathrm{~g})\end{array}$ & $\begin{array}{c}\mathrm{Mg}^{2+} \\
(\mathrm{meq} / 100 \mathrm{~g})\end{array}$ & $\begin{array}{c}\mathrm{MO} \\
(\%)\end{array}$ & $\begin{array}{c}\mathrm{CIC} \\
(\mathrm{Cmol} / \mathrm{kg})\end{array}$ & $\begin{array}{c}\mathrm{P} \\
(\mathrm{ppm})\end{array}$ & $\begin{array}{c}\mathrm{N} \\
(\%)\end{array}$ \\
\hline 1.12 & 28.98 & $\begin{array}{c}\text { Franco } \\
\text { arcillo } \\
\text { arenoso }\end{array}$ & 6.92 & 0.24 & 5.3 & 3.7 & 3.5 & 21.02 & 3.83 & 0.29 \\
\end{tabular}

Dap: densidad aparente, CRA: capacidad de retención de agua, CE: conductividad eléctrica, MO: materia orgánica, CIC: capacidad de intercambio catiónico. 
CUADRO II. MATRIZ DE TRATAMIENTOS.

\begin{tabular}{|c|c|c|c|c|c|}
\hline \multirow[t]{2}{*}{ Tratamiento } & \multirow[t]{2}{*}{ Descripción } & \multicolumn{2}{|c|}{ Biofertilizante (A) } & \multicolumn{2}{|c|}{ Fuente de nutrimentos (B) } \\
\hline & & $\begin{array}{l}\text { G. fasciculatum } \\
\text { (g/planta) }\end{array}$ & $\begin{array}{l}\text { B. subtilis } \\
\text { (mL/planta) }\end{array}$ & $\begin{array}{l}\text { Fertilizante químico } \\
\qquad(\mathrm{g} / \text { planta })\end{array}$ & $\begin{array}{c}\text { Abono orgánico } \\
\text { (g/planta) }\end{array}$ \\
\hline $\mathrm{T} 1$ & $\mathrm{~A}_{0} \mathrm{~B}_{0}$ & 0 & 0 & 0 & 0 \\
\hline $\mathrm{T} 2$ & $\mathrm{~A}_{0} \mathrm{~B}_{\mathrm{v}}$ & 0 & 0 & 0 & 31 \\
\hline $\mathrm{T} 3$ & $\mathrm{~A}_{0} \mathrm{~B}_{\mathrm{i}}$ & 0 & 0 & 4.24 & 0 \\
\hline $\mathrm{T} 4$ & $\mathrm{~A}_{\mathrm{m}} \mathrm{B}_{0}$ & 5 & 0 & 0 & 0 \\
\hline T5 & $\mathrm{A}_{\mathrm{m}} \mathrm{B}_{\mathrm{v}}$ & 5 & 0 & 0 & 31 \\
\hline T6 & $\mathrm{A}_{\mathrm{m}} \mathrm{B}_{\mathrm{i}}$ & 5 & 0 & 4.24 & 0 \\
\hline $\mathrm{T} 7$ & $\mathrm{~A}_{\mathrm{b}} \mathrm{B}_{0}$ & 0 & 2 & 0 & 0 \\
\hline $\mathrm{T} 8$ & $\mathrm{~A}_{\mathrm{b}} \mathrm{B}_{\mathrm{v}}$ & 0 & 2 & 0 & 31 \\
\hline T9 & $\mathrm{A}_{b} \mathrm{~B}_{\mathrm{i}}$ & 0 & 2 & 4.24 & 0 \\
\hline $\mathrm{T} 10$ & $\mathrm{~A}_{\mathrm{m}+\mathrm{b}} \mathrm{B}_{0}$ & 5 & 2 & 0 & 0 \\
\hline $\mathrm{T} 11$ & $\mathrm{~A}_{\mathrm{m}+\mathrm{b}} \mathrm{B}_{\mathrm{v}}$ & 5 & 2 & 0 & 31 \\
\hline $\mathrm{T} 12$ & $\mathrm{~A}_{\mathrm{m}+\mathrm{b}} \mathrm{B}_{\mathrm{i}}$ & 5 & 2 & 4.24 & 0 \\
\hline
\end{tabular}

m: G. fasciculatum; b: B. subtilis ; v: abono orgánico; i: fertilizante químico.

se realizó una comparación de medias con la prueba de la diferencia mínima significativa(DMS) a un nivel de significancia del $5 \%$. Las salidas se obtuvieron con el programa Statgraphics para Windows, versión 5.0.

\section{RESULTADOS Y DISCUSIÓN}

La altura máxima de la planta $(0.82 \mathrm{~m})$ se registró con la aplicación de abono orgánico (Fig. 1). El mejor crecimiento de la planta observado en este estudio podría atribuirse a la aplicación de abono orgánico como fuente de nutrimentos, el cual aumentó la cantidad y actividad de los microorganismos en el suelo. Geeta et al. (2016) realizaron un estudio sobre la influencia de NPK y biofertilizantes en el crecimiento, rendimiento y calidad del aster de China (Callistephus chinensis L.) cv. Poornima y revelaron que el crecimiento, rendimiento y calidad de la flor fueron influenciados significativamente por la apli-

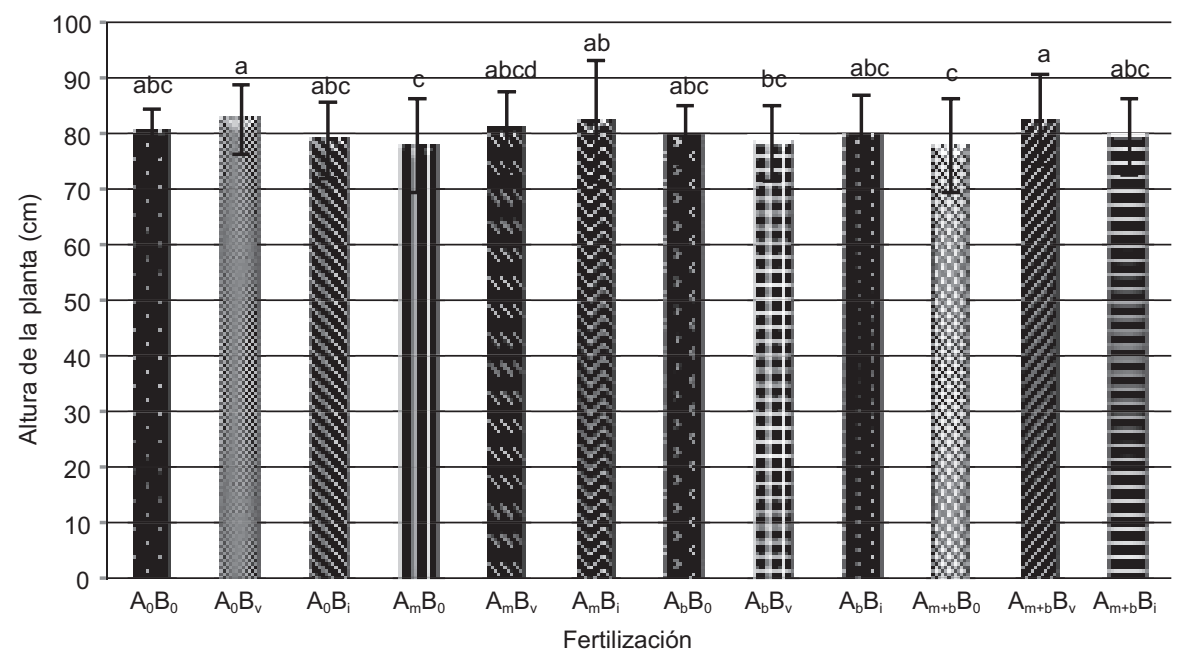

Fig. 1. Efecto de la fertilización (B) orgánica e inorgánica sobre la altura de plantas de gladiola (Gladiolus grandiflorus sp.) inoculadas con micorrizas $\left(\mathrm{A}_{\mathrm{m}}\right)$, Bacillus subtilis $\left(\mathrm{A}_{\mathrm{b}}\right)$, micorrizas y Bacillus subtilis $\left(\mathrm{A}_{\mathrm{m}+\mathrm{b}}\right)$. $\mathrm{B}_{\mathrm{o}}$ : sin fertilizar, $\mathrm{B}_{\mathrm{v}}$ : fertilizante orgánico, $\mathrm{B}_{\mathrm{i}}$ : fertilización con fertilizante inorgánico. Las letras diferentes en los histogramas indican diferencias significativas entre tratamientos de acuerdo con la prueba DMS $(\mathrm{P}<0.05)$. Los valores son medias $(\mathrm{n}=10)$. 


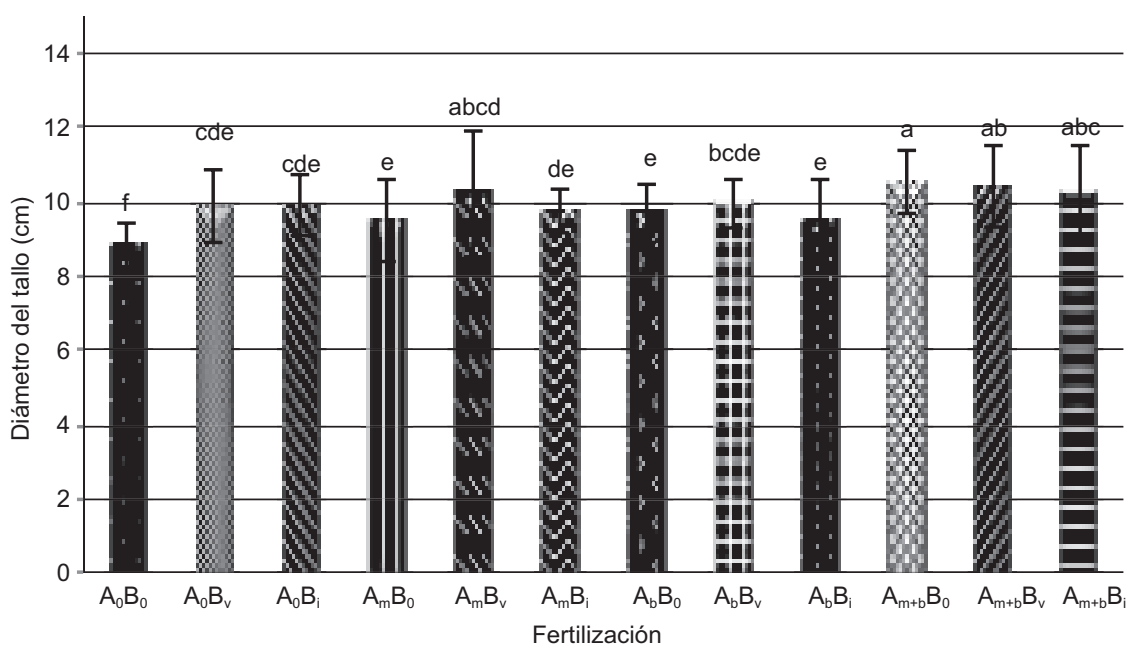

Fig. 2. Efecto de la fertilización (B) orgánica e inorgánica sobre el diámetro de tallo de plantas de gladiola (Gladiolus grandiflorus sp.) inoculadas con micorrizas $\left(\mathrm{A}_{\mathrm{m}}\right)$, Bacillus subtilis $\left(\mathrm{A}_{\mathrm{b}}\right)$, micorrizas y Bacillus subtilis $\left(\mathrm{A}_{\mathrm{m}+\mathrm{b}}\right)$. $\mathrm{B}_{\mathrm{o}}$ : $\sin$ fertilizar, $\mathrm{B}_{\mathrm{v}}$ : fertilizante orgánico, $\mathrm{B}_{\mathrm{i}}$ : fertilización con fertilizante inorgánico. Las letras diferentes en los histogramas indican diferencias significativas entre tratamientos de acuerdo con la prueba DMS $(\mathrm{P}<0.05)$. Los valores son medias $(\mathrm{n}=10)$.

cación combinada de biofertilizantes y diferentes niveles de fertilizantes inorgánicos.

Pansuriya y Chauhan (2015) investigaron el efecto sobre gladiola cv. Psittacinus Hybrid, de 20 t/ha de abono orgánico $+4 \mathrm{~kg} / \mathrm{ha}$ de Azotobacter $+4 \mathrm{~kg} / \mathrm{ha}$ de bacterias solubilizadoras de fósforo en comparación con fertilizante químico (NPK 300:150:150 kg/ha). La altura de la planta fue mayor en $5.81 \mathrm{~cm}$ en el tratamiento con abono orgánico y biofertilizantes en relación con el tratamiento donde se empleó fertilizante químico.

La inoculación con G. fasciculatum y B. subtilis originó un efecto positivo sobre el diámetro del tallo (Fig. 2). En un estudio realizado por Rubí et al. (2009) en lillium se observó un incremento en el diámetro del tallo cuando las plantas fueron inoculadas. Manoly et al. (2008) exploraron la posibilidad de cultivar Dahlia pinnata de forma no tradicional utilizando biofertilizantes (bacterias fijadoras de nitrógeno, solubilizadoras de fosfato y levaduras activas), accesibles económicamente para el productor y seguros para el ambiente; observaron que la biofertilización mixta incrementó en $17 \%$ el diámetro del tallo en dalia.

La inoculación con G. fasciculatum más abono orgánico incrementó el peso fresco de las plantas de gladiola (Fig. 3), resultados que coinciden con los de Pansuriya y Chauhan (2015), quienes evaluaron el crecimiento de gladiola cv. Psittacinus Hybrid; el peso fresco de la planta fue superior cuando se utilizó abono orgánico, Azotobacter y bacterias solubilizadoras de fósforo en comparación con fertilizantes químicos.

El peso seco de la parte aérea (Fig. 4) fue significativamente mayor cuando se aplicó $B$. subtilis más abono orgánico. Resultados similares fueron encontrados por Chang et al. (2010), quienes compararon fertilizantes inorgánicos y orgánicos en Anthurium andreanum y detectaron que el peso seco de planta más alto se registró con abono orgánico. El mayor peso seco cuando se usa fertilizante orgánico puede deberse a su alto contenido de materia orgánica, el cual tiende a mejorar las propiedades físicas, químicas y biológicas del suelo, lo que favorece el crecimiento de las plantas. En Dahlia pinnata el peso seco de la planta aumentó significativamente (31\%) (Manoly et al. 2008). En gladiola, Pansuriya y Chauhan (2015) encontraron una diferencia de 4.01 g al usar abono orgánico vs. fertilizante químico.

En gladiola, la inoculación con $G$. fasciculatum $\mathrm{y}$ abono orgánico $\left(\mathrm{A}_{\mathrm{m}} \mathrm{B}_{\mathrm{v}}\right)$ incrementó el número de flores (Fig. 5); la inoculación con HMA tuvo una respuesta positiva. Abdou et al. (2013) estudiaron en Gladiolus grandiflorus el efecto de la combinación de biofertilizantes (microorganismos efectivos y levaduras activas) con composta y observaron un incrementó en el número de flores. Gaur et al. (2000) estudiaron el desarrollo de tres plantas ornamentales inoculadas con HMA y reconocieron una mejoría 


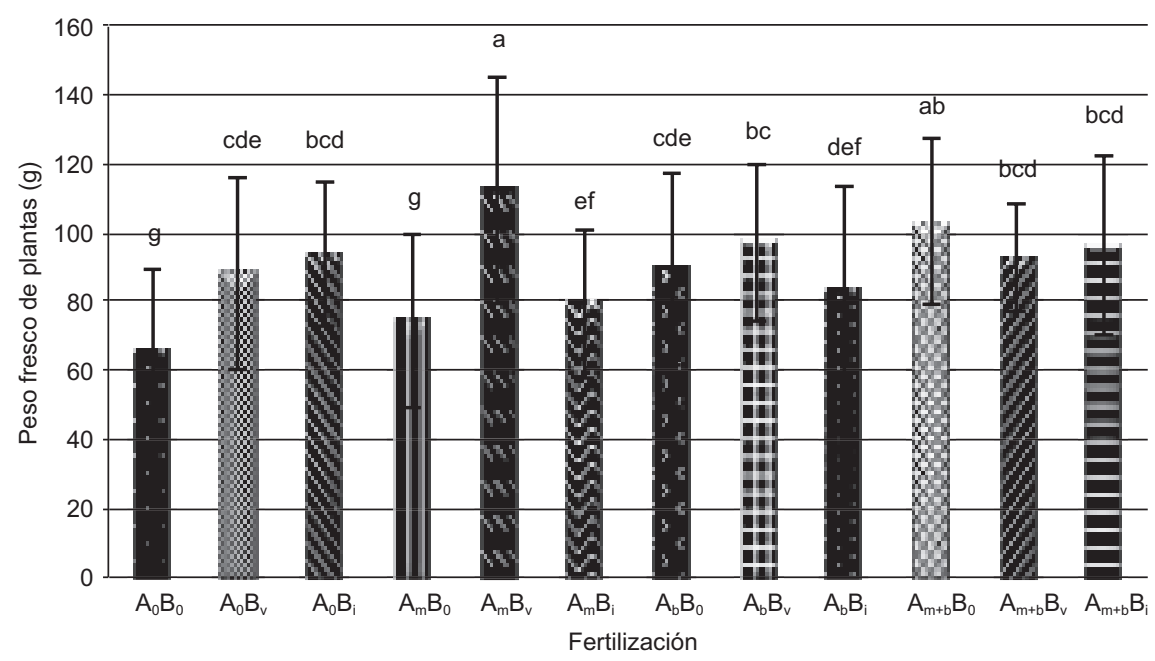

Fig. 3. Efecto de la fertilización (B) orgánica e inorgánica sobre el peso fresco de plantas de gladiola (Gladiolus grandiflorus sp.) inoculadas con micorrizas $\left(\mathrm{A}_{\mathrm{m}}\right)$, Bacillus subtilis $\left(\mathrm{A}_{\mathrm{b}}\right)$, micorrizas y Bacillus subtilis $\left(\mathrm{A}_{\mathrm{m}+\mathrm{b}}\right)$. $\mathrm{B}_{\mathrm{o}}$ : sin fertilizar, $\mathrm{B}_{\mathrm{v}}$ : fertilizante orgánico, $B_{i}$ : fertilización con fertilizante inorgánico. Las letras diferentes en los histogramas indican diferencias significativas entre tratamientos de acuerdo con la prueba DMS $(\mathrm{P}<0.05)$. Los valores son medias $(\mathrm{n}=10)$.

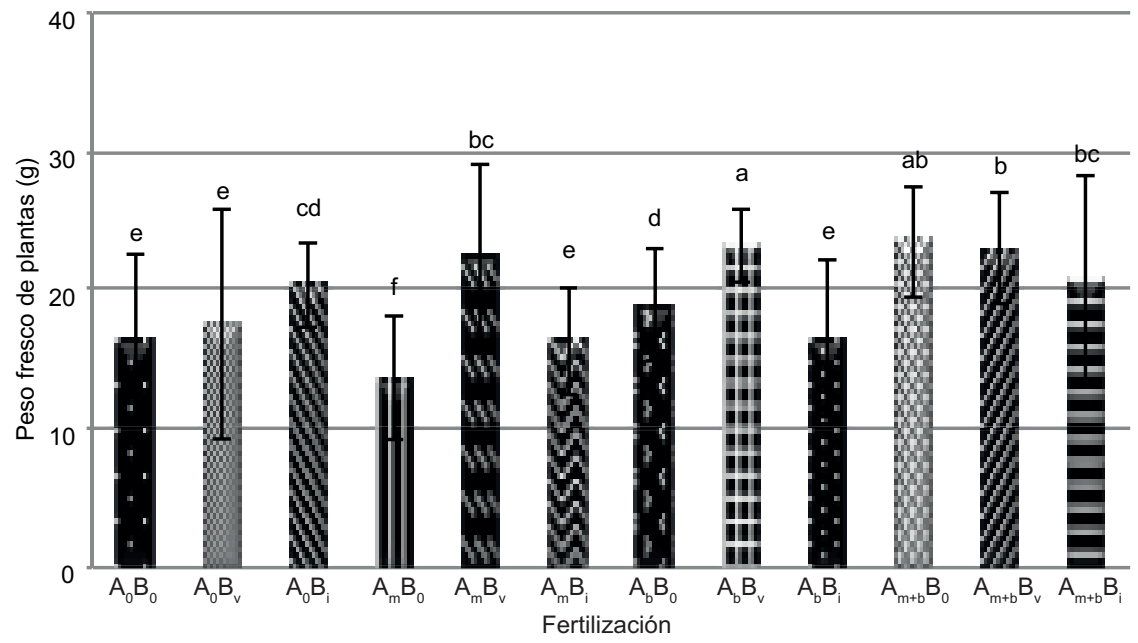

Fig. 4. Efecto de la fertilización (B) orgánica e inorgánica sobre el peso seco de plantas de gladiola (Gladiolus grandiflorus sp.) inoculadas con micorrizas $\left(\mathrm{A}_{\mathrm{m}}\right)$, Bacillus subtilis $\left(\mathrm{A}_{\mathrm{b}}\right)$, micorrizas y Bacillus subtilis $\left(\mathrm{A}_{\mathrm{m}+\mathrm{b}}\right)$. $\mathrm{B}_{\mathrm{o}}$ : $\sin$ fertilizar, $\mathrm{B}_{\mathrm{v}}$ : fertilizante orgánico, $\mathrm{B}_{\mathrm{i}}$ : fertilización con fertilizante inorgánico. Las letras diferentes en los histogramas indican diferencias significativas entre tratamientos de acuerdo con la prueba DMS $(\mathrm{P}<0.05)$. Los valores son medias $(\mathrm{n}=10)$.

en los parámetros vegetativo y reproductivo. Rao et al. (2015) llevaron a cabo un experimento para estudiar la influencia de las prácticas de manejo integrado de nutrientes para mejorar el crecimiento y el rendimiento de tuberosa (Polianthes tuberosa L.) cv. Hyderabad Doublee. La combinación de abonos orgánicos y biofertilizantes favorecieron la expresión de un mayor número de flores por espiga.

Rao et al. (2015), estudiaron la influencia de prácticas de gestión integrada de nutrientes, para mejorar el crecimiento de la flor de nardo cv. Hyderabad Double. Los tratamientos constaron de abonos orgánicos y 


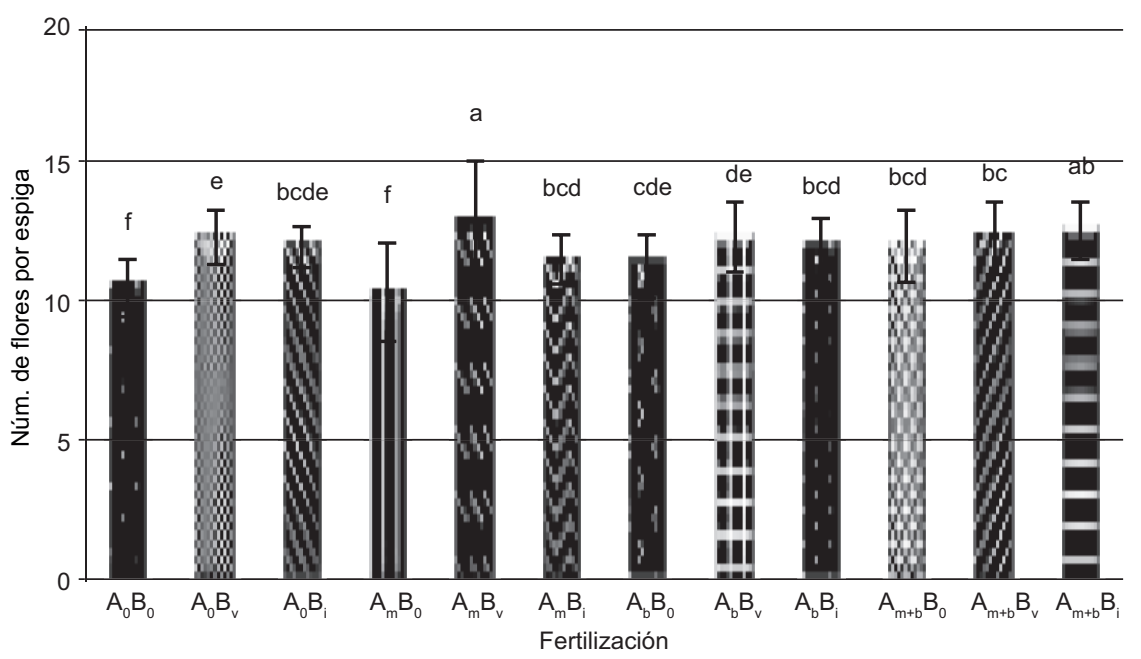

Fig. 5. Efecto de la fertilización (B) orgánica e inorgánica sobre flores por espiga de plantas de gladiola (Gladiolus grandiflorus sp.) inoculadas con micorrizas $\left(\mathrm{A}_{\mathrm{m}}\right)$, Bacillus subtilis $\left(\mathrm{A}_{\mathrm{b}}\right)$, micorrizas y Bacillus subtilis $\left(\mathrm{A}_{\mathrm{m}+\mathrm{b}}\right) . \mathrm{B}_{\mathrm{o}}$ : $\sin$ fertilizar, $\mathrm{B}_{\mathrm{v}}$ : fertilizante orgánico, $\mathrm{B}_{\mathrm{i}}$ : fertilización con fertilizante inorgánico. Las letras diferentes en los histogramas indican diferencias significativas entre tratamientos de acuerdo con la prueba DMS $(\mathrm{P}<0.05)$. Los valores son medias $(\mathrm{n}=10)$.

biofertilizantes más diferentes niveles de NPK $(100,75$ y $50 \%$ de la dosis de fertilización recomendada). Con estiércol + vermicomposta + Azospirillum $2 \mathrm{~g} /$ planta y bacterias solubilizadoras de fósforo ( $2 \mathrm{~g} / \mathrm{planta}) \mathrm{se}$ favoreció un mayor número de flores por espiga.

$\mathrm{Al}$ inocular los cormos de gladiola con G. fasciculatum hubo un incremento significativo del contenido de clorofila (Fig. 6) y, en consecuencia, de la cantidad de nitrógeno en las hojas. G. fasciculatum + abono orgánico tuvieron efectos significativos $(\mathrm{p} \leq 0.05)$ en el contenido de clorofila (11.51 unidades spad más que el tratamiento testigo) (Fig. 6).

El diámetro significativamente mayor $(50.28 \mathrm{~mm})$ del cormo se registró cuando se aplicó G. fasciculatum + abono orgánico; en este tratamiento el peso del cormo fuemayor en $24.07 \mathrm{~g}$ con relación al testigo (Cuadro III).

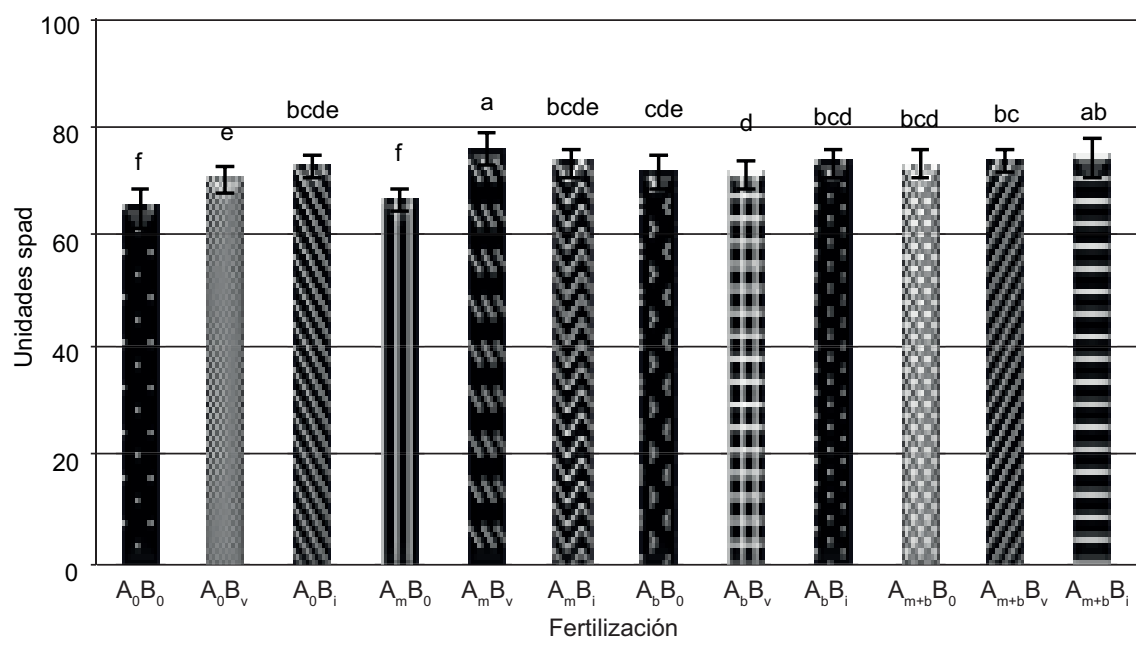

Fig. 6. Efecto de la fertilización (B) orgánica e inorgánica sobre las unidades spad en plantas de gladiola (Gladiolus grandiflorus sp.) inoculadas con micorrizas $\left(\mathrm{A}_{\mathrm{m}}\right)$, Bacillus subtilis $\left(\mathrm{A}_{\mathrm{b}}\right)$, micorrizas y Bacillus subtilis $\left(\mathrm{A}_{\mathrm{m}+\mathrm{b}}\right)$. $\mathrm{B}_{\mathrm{o}}$ : sin fertilizar, $\mathrm{B}_{\mathrm{v}}$ : fertilizante orgánico, $\mathrm{B}_{\mathrm{i}}$ : fertilización con fertilizante inorgánico. Las letras diferentes en los histogramas indican diferencias significativas entre tratamientos de acuerdo con la prueba DMS $(\mathrm{P}<0.05)$. Los valores son medias $(\mathrm{n}=10)$. 
CUADRO III. EFECTO DE LA INOCULACIÓN DE GLADIOLA CON Glomus fasciculatum Y Bacillus subtilis SOBRE CORMOS Y CORMILLOS.

\begin{tabular}{lcccc}
\hline Tratamiento & \multicolumn{4}{c}{ Variable } \\
\cline { 2 - 5 } & $\begin{array}{c}\text { Diámetro del } \\
\text { cormo }(\mathrm{mm})\end{array}$ & $\begin{array}{c}\text { Peso del } \\
\text { cormo }(\mathrm{g})\end{array}$ & $\begin{array}{c}\text { Cormillos } \\
\text { por planta }\end{array}$ & $\begin{array}{c}\text { Peso de los } \\
\text { cormillos }(\mathrm{g})\end{array}$ \\
\hline $\mathrm{A}_{0} \mathrm{~B}_{0}$ & $39.28^{\mathrm{d}}$ & $21.88^{\mathrm{e}}$ & $49.5^{\mathrm{d}}$ & $16.05^{\mathrm{d}}$ \\
$\mathrm{A}_{0} \mathrm{~B}_{\mathrm{v}}$ & $48.37^{\mathrm{abc}}$ & $37.46^{\mathrm{bcd}}$ & $58^{\mathrm{c}}$ & $20.93^{\mathrm{c}}$ \\
$\mathrm{A}_{0} \mathrm{~B}_{\mathrm{i}}$ & $46.11^{\mathrm{c}}$ & $38.13^{\mathrm{bcd}}$ & $45.27^{\mathrm{b}}$ & $18.10^{\mathrm{e}}$ \\
$\mathrm{A}_{\mathrm{m}} \mathrm{B}_{0}$ & $45.99^{\mathrm{c}}$ & $42.97^{\mathrm{abc}}$ & $31.41^{\mathrm{e}}$ & $15.79^{\mathrm{d}}$ \\
$\mathrm{A}_{\mathrm{m}} \mathrm{B}_{\mathrm{v}}$ & $50.28^{\mathrm{a}}$ & $42.18^{\mathrm{abc}}$ & $74.5^{\mathrm{b}}$ & $28.03^{\mathrm{b}}$ \\
$\mathrm{A}_{\mathrm{m}} \mathrm{B}_{\mathrm{i}}$ & $47.90^{\mathrm{abc}}$ & $36.63^{\mathrm{bcd}}$ & $48.22^{\mathrm{c}}$ & $16.15^{\mathrm{d}}$ \\
$\mathrm{A}_{\mathrm{b}} \mathrm{B}_{0}$ & $47.35^{\mathrm{bc}}$ & $33.98^{\mathrm{d}}$ & $69.66^{\mathrm{b}}$ & $26.81^{\mathrm{b}}$ \\
$\mathrm{A}_{\mathrm{b}} \mathrm{B}_{\mathrm{v}}$ & $50.33^{\mathrm{a}}$ & $45.81^{\mathrm{a}}$ & $35.83^{\mathrm{d}}$ & $10.75^{\mathrm{f}}$ \\
$\mathrm{A}_{\mathrm{b}} \mathrm{B}_{\mathrm{i}}$ & $48.26^{\mathrm{abc}}$ & $35.82^{\mathrm{cd}}$ & $70.66^{\mathrm{c}}$ & $28.33^{\mathrm{b}}$ \\
$\mathrm{A}_{\mathrm{m}+\mathrm{b}} \mathrm{B}_{0}$ & $48.15^{\mathrm{abc}}$ & $43.38^{\mathrm{ab}}$ & $45.27^{\mathrm{c}}$ & $18.10^{\mathrm{d}}$ \\
$\mathrm{A}_{\mathrm{m}+\mathrm{b}} \mathrm{B}_{\mathrm{v}}$ & $49.66^{\mathrm{ab}}$ & $45.53^{\mathrm{a}}$ & $87.66^{\mathrm{a}}$ & $30.51^{\mathrm{a}}$ \\
$\mathrm{A}_{\mathrm{m}+\mathrm{b}} \mathrm{B}_{\mathrm{i}}$ & $45.93^{\mathrm{c}}$ & $32.04^{\mathrm{d}}$ & $48.27^{\mathrm{c}}$ & $13.96^{\mathrm{e}}$ \\
\hline
\end{tabular}

Letras iguales en la misma columna indican valores estadísticamente semejantes $(\mathrm{p} \leq 0.05)$.

$\mathrm{A}_{0}$ : sin inoculación; $\mathrm{A}_{\mathrm{m}}$ : inoculación con micorrizas; $\mathrm{A}_{\mathrm{b}}$ : inoculación con Bacillus subtilis; $\mathrm{A}_{\mathrm{m}+\mathrm{b}}$ : coinoculación (micorrizas + Bacillus Subtilis); $\mathrm{B}_{0}$ : sin fertilización; $\mathrm{B}_{\mathrm{v}}$ : abono orgánico; $\mathrm{B}_{\mathrm{i}}$ : fertilizante inorgánico.

Lo anterior puede deberse a la mayor cantidad de nutrientes disponibles por efecto de la combinación de biofertilizantes y abono orgánico. Satapathy et al. (2016) estudiaron el impacto del manejo integrado de nutrientes sobre la floración y la producción de cormos en gladiola; sus resultados mostraron que con $75 \%$ de la dosis recomendada de fertilizantes, vermicomposta en 5 t/ha y Azospirrillum + bacterias solubilizadoras de fósforo se favoreció un aumento de peso $(75.66 \mathrm{~g})$ y diámetro $(0.065 \mathrm{~m})$ en el cormo hijo, así como en el número (58.36) y peso (32.43 g) de los cormillos por planta. El aumento del diámetro y peso del cormo podría deberse a la mayor disponibilidad de nutrientes para la planta por la acción de los biofertilizantes y a un mayor aporte de materia orgánica por parte del abono orgánico. Se incrementó la actividad fotosintética de las plantas y el transporte de nutrientes hacia el cormo. Baskaran et al. (2014) encontraron que el número máximo de cormos por planta fue producido por Azospirillum (2.9), seguido de bacterias solubilizadoras de fósforo. Esto puede deberse a una mayor acumulación de asimilados en los cormos en desarrollo.

\section{CONCLUSIONES}

La aplicación de G. fasciculatum y abono orgánico favoreció el diámetro del tallo y el número de flores, variables importantes para la comercialización de la gladiola.

Con relación al cormo, el mejor tratamiento fue G. fasciculatum + B. subtilis + abono orgánico, el cual superó al control con incrementos de $27 \%$ en diámetro y $100 \%$ en peso; además, mejoró en $77 \%$ el número y en $90 \%$ el peso de cormillos, parámetros altamente valorados por los productores dado que garantizan material para la reproducción de gladiola con calidad adecuada.

G. fasciculatum + B. subtilis + abono orgánico ejercen un efecto positivo en el crecimiento vegetativo y reproductivo de las plantas de gladiola, por lo que representan una alternativa de manejo sostenible de esta especie, que favorece la disminución del uso de fertilizantes químicos y los daños a la salud humana $\mathrm{y}$ al ambiente.

\section{REFERENCIAS}

Abdou M.A.H., Aly M.K. y Ahmed A.S.A. (2013). Effect of compost, biofertilization and some vitamins addition on Gladiolus grandiflorus. J. Plant Prod. 4 (12), 1751-1761. https://doi.org/10.21608/ jpp.2013.75096

Alam M., Khaliq A., Sattar A., Shukla R.S., Anwar M. y Dharni S. (2011). Synergistic effect of arbuscular 
mycorrhizal fungi and Bacillus subtilis on the biomass and essential oil yield of rose-scented geranium (Pelargonium graveolens). Arch. Agron. Soil Sci. 57 (8), 889-898. https://doi.org/10.1080/03650340.201 0.498013

Astier-Calderón M., Maass-Moreno M. y Etchevers-Barra J. (2002). Derivación de indicadores de calidad de suelos en el contexto de la agricultura sustentable. Agrociencia 36 (5), 605-620.

Baskaran V., Misra R.L., Singh S.K. y Abirami K. (2014). Response of bio-fertilizers and commercial formulations on growth, yield and corm production of gladiolus. Indian J. Hort. 71 (2), 237-241.

Cantor M. y Tolety J. (2011). Gladiolus. En: Wild crop relatives: Genomic and breeding resources. plantation and ornamental crops (Kole C., Ed.). Springer Heidelberg, Alemania, 133-159. https://doi.org/10.1007/9783-642-21201-7

Chang K.H., Wu R.Y., Chuang K.C., Hsieh T.F. y Chung R.S. (2010). Effects of chemical and organic fertilizers on the growth, flower quality and nutrient uptake of Anthurium andreanum, cultivated for cut flower production. Sci. Hortic. 125 (3), 434-441. https://doi. org/10.1016/j.scienta.2010.04.011

Da Silva T., Estebanez R., do Carmo B., Rossetti, J., Montezano, J., Martins M., de Vasconcelos, F., Sales, C. y Seldin, L. (2017). Cultivable bacterial communities associated with roots of rose-scented geranium (Pelargonium graveolens) with the potential to contribute to plant growth. App. Soil Ecol. 111, 123-128 https://doi.org/10.1016/j.apsoil.2016.12.002.

D’Hose T., Cougnon M., Vliegher A., Vandecasteele B., Viaene N., Cornelis W., Bockstaele E.V. y Reheul D. (2014). The positive relationship between soil quality and crop production: a case study on the effect of farm compost application. Appl. Soil Ecol. 75, 189-198. https://doi.org/10.1016/j.apsoil.2013.11.013

Dipanjali B., Madhumita T. y Vikash K. (2018). Effect of organic manures and biofertilizers on growth and yield of gladiolus (Gladiolus grandiflorus L.). Int. J. Chem. Stud. 6 (5), 2529-2532. https://doi.org/10.13140/ RG.2.2.16257.30561

García E. (2004). Modificaciones al sistema de clasificación climática de Köppen. $5 \mathrm{a}$ ed. Instituto de Geografía, UNAM, Ciudad de México, 91 pp.

Gaur A., Gaur A. y Adholeya A. (2000). Growth and flowering in Petunia hybrida, Callistephus chinensis and Impatiens balsamina inoculated with mixed AM inocula or chemical fertilizers in a soil of low $P$ fertility. Sci. Hortic. 84 (1-2), 151-162. https://doi.org/10.1016/ s0304-4238(99)00105-3

Geeta B.L., Siddappa, Mahadevamma M., Raj L. y Totad M. (2016). Influence of NPK and biofertilizers on growth, yield and quality of china aster (Callistephus chinensis L. Nees) for cut flower production. Res. Environ. Life Sci. 9 (10), 1236-1238.

González-Pérez E., Ayala-Garay O.J., Carrillo-Salazar J.A., García-de Los Santos G., Yáñez- Morales M.J. y Juárez-Muñoz J. (2011). Estudio del desarrollo, calidad de flor y dosis de fertilización en gladiolo (Gladiolus grandiflorus Hort.). Rev. Fitotec. Mex. 34 (4), 277-283.

Granatstein D. y Kupferman E. (2008). Sustainable horticulture in fruit production. Acta Hortic. 767, 295-308. https://doi.org/10.17660/ActaHortic.2008.767.31

Hashem A., Tabassum B. y Abd E. (2019). Bacillus subtilis: a plant-growth promoting rhizobacterium that also impacts biotic stress. Saudi J. Biol. Sci. 26 (6), 1291-1297. https://doi.org/10.1016/j.sjbs.2019.05.004.

Hayek S., Grosch R., Gianinazzi-Pearson V. y Franken P. (2012). Bioprotection and alternative fertilisation of petunia using mycorrhiza in a soilless production system. Agron. Sustain. Dev. 32 (3), 765-771. https:// doi.org/10.1007/s13593-012-0083-z

Kholkute R. (2013). Biofertilizers: Opportunities and challenges. Arab Fertilizer Associations Magazine 65, 40-43.

Lee E.H., Eo J.K., Ka K.H. y Eom A.H. (2013). Diversity of arbuscular mycorrhizal fungi and their roles in ecosystems. Mycobiology 41 (3), 121-125. https://doi. org/10.5941/MYCO.2013.41.3.121

López-Vald'ss F., Fernández-Luqueño F., CeballosRamírez J.M., Marsch R., Olalde-Portugal V. y Dendooven L. (2011). A strain of Bacillus subtilis stimulates sunflower growth (Helianthus annus L.) temporarily. Scientia Horticulturae 128 (4), 499-505. https://doi.org/10.1016/j.scienta.2011.02.006

Manoly D.N. y Nasr A. (2008). Response of Dahlia pinnata plants to biofertilizer types. Egypt. J. Exp. Biol. Bot. 4, 87-91.

Pansuriya P.B. y Chauhan R.V. (2015). Effect of integrated nutrient management on growth, yield and quality of gladiolus (Gladiolus grandiflorus L.) $\mathrm{Cv}$. Psittacinus Hybrid. J. Hortic. 2 (2), 129. https://doi. org/10.4172/2376-0354.1000129

Parlakova F., Dursun A., Tekiner N., Kul R. y Kotan R. (2019). Efficacy of vermicompost and/or plant growth and development in gladiolus. Ornam. Hortic. 25 (2), 180-188. https://doi.org/10.14295/oh.v25i2.2023

Parniske M. (2008). Arbuscular mycorrhiza: the mother of plant root endosymbioses. Nature Reviews Microbiology 6 (10), 763-775. https://doi.org/10.1038/ nrmicro1987

Pérez-García A. y Romero D. (2011). Plant protection and growth stimulation by microorganisms: biotechnological applications of Bacilli in agriculture. Curr. Opin. Biotech. 22 (2), 187-193. https://doi.org/10.1016/j. copbio.2010.12.003 
Pullman M.E., Maloni M.J. y Carter C.R. (2009). Food for thought: social versus environmental sustainability practices and performance outcomes. J. Supply Chain Manag. 45 (4), 38-54. https://doi.org/10.1111/j.1745493X.2009.03175.x

Püschel D., Rydlová J. y Vosátka M. (2014). Can mycorrhizal inoculation stimulate the growth and flowering of peat-grown ornamental plants under standard or reduced watering? Appl. Soil Ecol. 80, 93-99. https:// doi.org/10.1016/j.apsoil.2014.04.001

Qasim M., Younis A., Zahir A., Riaz H. y Tariq U. (2014). Microbial inoculation increases the nutrient A. uptake efficiency for quality production of Gladiolus grandiflorus. Pak. J. Agri. Sci. 51 (4), 875-880.

Rao K., Kameswari P. y Rani T. (2015). Impact of integrated nutrient management on growth, flowering, yield, and economics of tuberose. Agricultural Science Digest-A Research Journal 35 (1), 66-69. https://doi. org/10.5958/0976-0547.2015.00014.2

Roger-Estrade J., Anger C., Bertrand M. y Richard G. (2010). Tillage and soil ecology: partners for sustainable agriculture. Soil and Tillage Research 111 (1), 33-40. https://doi.org/10.1016/j.still.2010.08.010

Rubí M., Olalde V., Reyes B.G., González A. y Aguilera L.I. (2009). Influencia de Glomus fasciculatum en el crecimiento y desarrollo de Lilium sp. cv orange pixie. Agricultura Técnica en México 35 (2), 201-210.

Sahle A. y Potting J. (2013). Environmental life cycle assessment of Ethiopian rose cultivation. Sci. Total Environ. 443 (15), 163-172. https://doi.org/10.1016/j. scitotenv.2012.10.048

Satapathy S.P., Toppo R., Dishri M. y Mohanty C.R. (2016). Impact of integrated nutrient management (INM) on flowering and corm production in gladiolus. Biom. Biostat. Int. J. 4 (7), 296-298. https://doi. org/10.15406/bbij.2016.04.00119

Schwab N.T., Streck N.A., Becker C.C., Langner J.A., Uhlmann L.O. y Ribeiro B.S.M.R. (2015). A phenological scale for the development of Gladiolus. Ann. Appl. Biol. 166 (3), 496-507. https://doi.org/10.1111/ aab.12198
Shanmugam V., Kanoujia N., Singh M., Singh S. y Prasad R. (2011). Biocontrol of vascular wilt and corm rot of gladiolus caused by Fusarium oxysporum f. sp. gladioli using plant growth promoting rhizobacterial mixture. Crop Prot. 30 (7), 807-813. https://doi.org/10.1016/j. cropro.2011.02.033

Sharma A., Saha T.N., Arora A., Shah R. y Nain L. (2017). Efficient microorganism compost benefits plant growth and improves soil health in calendula and marigold. Horticultural Plant Journal 3 (2), 67-72. https://doi. org/10.1016/j.hpj.2017.07.003

Sharma, S., Padbhushan R. y Kumar U. (2019). Integrated nutrient management in rice-wheat cropping system: an evidence on sustainability in the Indian subcontinent through meta-analysis. Agronomy 9 (2), 71-85. https:// doi.org/103390/agronomy9020071

SIAP (2017). Anuario del Servicio de Información Agroalimentaria y Pesquera, ciclo 2016. Secretaria de Agricultura, Ganadería, Desarrollo Rural, Pesca y Alimentación [en línea]. https://nube.siap.gob.mx/ cierreagricola/ 03/12/2017.

Smith S.E. y Read D. (2008). Mycorrhizal symbiosis. 3a ed. Academic Press. New York, EUA, 800 pp.

Valdez-Aguilar, L.A., Hernández-Pérez, A., AlvaradoCamarillo, D. y Cruz-Altunar A. (2015). Diseño de un programa de fertilización para crisantemo en base a extracción de macronutrimentos. Rev. Mexicana Cienc. Agric. 6 (12), 2263-2276.

Verbruggen E., van Der Heijden M.G.A., Weedon J.T., Kowalchuk G.A. y Röling W.F.M. (2012). Community assembly, species richness and nestedness of arbuscular mycorrhizal fungi in agricultural soils. Molecular Ecology 21 (10), 2341-2353. https://doi. org/10.1111/j.1365-294X.2012.05534.x 